\title{
A New Method for Color Image Quality Assessment
}

\author{
Niveditta Thakur \\ Student, ECE Department \\ NITTTR \\ Chandigarh-160019, India
}

\author{
Swapna Devi \\ Associate Professor, ECE Department \\ NITTTR \\ Chandigarh-160019, India
}

\begin{abstract}
Humans have always seen the world in color. In the last three decades, there has been rapid and enormous transition from grayscale images to color ones. Well-known objective evaluation algorithms for measuring image quality include mean squared error (MSE), peak signal-to-noise ratio (PSNR), and human Visual System based one are structural similarity measures and edge based similarity measures. One of the common and major limitations of these objective measures is that they evaluate the quality of grayscale images only and don't make use of image color information. Since, Color is a powerful descriptor that often simplifies the object identification and extraction from a scene so color information also could influence human beings' judgments. So, in this paper new objective color image quality measure in spatial domain is proposed that overcomes the limitation of these existing methods significantly, is easy to calculate and applicable to various image processing applications. The proposed quality measure has been designed as a combination of four main factors: luminance similarity, structure correlation, edge similarity, and color similarity. This proposed index is mathematically defined and in it HVS model is explicitly employed. Experiments on various image distortion types indicate that this index performs significantly better than other traditional error summation methods and existing similarity measures.
\end{abstract}

\section{Keywords}

Human visual system (HVS), image quality assessment (IQA), mean structural similarity index (MSSIM), mean squared error (MSE), Visual Information Fidelity in Pixel Domain (VIFP), peak signal to noise ratio (PSNR).

\section{INTRODUCTION}

There has been explosive growth in the use of multimedia technologies and applications. In the last three decades, there has been rapid and enormous transition from grayscale images to color ones. We are exposed to color images on a daily basis in print, photographs, television, computer displays, and cinema movies, where color plays a vital role. Color monitors, printers, and copiers now dominate the office and home environments. With this rapid progression, color and multispectral properties of images are becoming increasingly crucial to the field of image processing, often extending and/or replacing previously known grayscale techniques. Color has been widely utilized and exploited for its properties in many applications, including multimedia and video, database indexing and retrieval, and exchange of information. Thus, color images are more widely used for information representation and communication. But unfortunately, these images are subjected to wide variety of distortions during its acquisition, subsequent compression, transmission, processing and then reproduction, which degrade visual quality. So, measurement of image quality is crucial for many image processing systems.
There are two ways of measuring image quality which are subjective and objective [1]. In subjective measure, quality is judged by group of human observers, which is usually inconvenient, time-consuming and expensive. Objective measures, on other hand are quantitative measures that can automatically predict the perceived image quality. Objective image quality metrics can be classified to full reference (FR), no reference (NR) and reduced reference (RR). Full reference image quality assessment (FR-IQA) demands that a complete reference image is to be known, while no reference image quality assessment (NR-IQA) means that the reference image is not available. In the third type, the reference image is only partially available, and is in the form of a set of extracted features. This is referred to as reduced reference image quality assessment (RR-IQA). This paper presents a full reference image quality assessment method for color images where original test image is available.

Objective image quality assessments can be divided into different categories [2]. The first category includes difference based measures like mean squared error (MSE) and the peak signal-to-noise ratio (PSNR); the second category is human visual system (HVS) based that includes structure similarity measures like mean structural similarity index ( MSSIM) [6], visual information fidelity in pixel domain (VIFP), structure \& hue similarity (SHSIM) [12], and edge similarity based measures. The HVS based methods take advantage of the known characteristics of HVS [11], and measure image quality by estimating perceived errors. MSSIM fail on particular image impairments like in case of highly blurred and Gaussian contaminated images. Existing edge based structural similarity measures overcome the limitations of structural similarity index but not up to full extent showing the inconsistency with HVS. Also, most of these objective measures evaluate the quality of grayscale images only and don't make use of image color information.

In this paper, HVS based objective image quality index $\left(\mathrm{Q}_{\text {new }}\right)$ is developed which overcomes the limitation of existing methods and takes in to account parameters like structure, luminance, edge and color. Parameters are selected based on the fact that they must be sensitive to distortions. Section 2 describes the existing methods and their limitations followed by section 3 which is highlights color image fundamentals, Section 4 discusses the formulation of the proposed index which is based on structure, edge, color and luminance similarity followed by section 5 showing implementation and analysis of results. Finally, section 6 gives the conclusion of the paper.

\section{BACKGROUND}

Most widely used image quality metrics viz., MSE, PSNR, and SSIM are discussed. MSE and PSNR are difference based measures whereas structural similarity measures are human visual system based one. 


\subsection{MSE/PSNR}

The simplest and most widely used fidelity measure is the mean squared error (MSE) and the corresponding distortion metric, the peak signal-to-noise ratio (PSNR) [3],[4] is given by:

$$
\begin{aligned}
& M S E=\frac{1}{M N} \sum_{m=1}^{M} \cdot \sum_{n=1}^{N}\left[f(m, n)-f^{\prime}(m, n)\right]^{2} \\
& P S N R=10 \log _{10} \frac{255^{2}}{M S E}
\end{aligned}
$$

Due to its evident physical meanings and mathematical convenience this is widely used. But it is not very well matched to human judgment of image quality.

\subsection{Universal Image Quality Index (UQI)}

Instead of using traditional error summation methods, Wang and Bovik proposed a method to model any image distortion via a combination of three factors: loss of correlation, luminance distortion, and contrast distortion and named it as Universal Quality Index (UQI) [5].

Let, $X=\left\{x_{i} \mid i=1,2, \ldots N\right\}$ and $Y=\left\{y_{i} \mid i=1,2, \ldots N\right\}$ be the original and test image signal respectively. If $\bar{x}$ is the mean of $\mathrm{x}, \sigma_{x}^{2}$, the variance of $\mathrm{x}, \sigma_{x y}$ is covariance of $\mathrm{x}, \mathrm{y}$, then, UQI is given by:

$$
\begin{gathered}
U Q I=\frac{4 \sigma_{x y} \overline{x y}}{\left(\bar{x}^{2}+\bar{y}^{2}\right)\left(\sigma_{x}{ }^{2}+\sigma_{y}{ }^{2}\right)} \\
\text { where, } \quad \bar{x}=\frac{1}{N} \sum_{i}^{N} x_{i} \text { and, } \bar{y}=\frac{1}{N} \sum_{i}^{N} y_{i}
\end{gathered}
$$

Also, standard deviation given as

$$
\begin{aligned}
\sigma_{x} & =\sqrt{\frac{1}{N-1}\left[\sum_{i=1}^{N}\left(x_{i}-\mu_{x}\right)^{2}\right]} \text { and, } \\
\sigma_{y} & =\sqrt{\frac{1}{N-1}\left[\sum_{i=1}^{N}\left(y_{i}-\mu_{y}\right)^{2}\right]}
\end{aligned}
$$

covariance is given as

$$
\sigma_{x y}=\frac{1}{N-1} \sum_{i=1}^{N}\left(x_{i}-\mu_{x}\right)\left(y_{i}-\mu_{y}\right)
$$

The dynamic range of UQI is $[0,1]$. The best value of 1 is achieved if and only if $\mathrm{y}_{\mathrm{i}}=\mathrm{x}_{\mathrm{i}}$ for all $\mathrm{i}=1,2, \ldots \ldots . \mathrm{N}$.

$$
U Q I=\frac{\sigma_{x y}}{\sigma_{x} \sigma_{y}} \frac{2 \overline{x y}}{\left(\bar{x}^{2}+\bar{y}^{2}\right)} \frac{2 \sigma_{x} \sigma_{y}}{\left(\sigma_{x}^{2}+\sigma_{y}^{2}\right)}
$$

This quality index models any distortion as a combination of three different factors as shown clearly in eq. 4: loss of correlation as represented by the first term where second component represents luminance distortion and the last component is contrast distortion. Thus, UQI can be written as a product of three components.

\subsection{Mean Structural Similarity Index}

The principal idea underlying the structural similarity approach as proposed by Zhou Wang [6] is that the HVS is highly adapted to extract structural information from visual scenes. Structural similarity index (SSIM) includes three parts luminance comparison, contrast comparison, and structure comparison $\mathrm{s}(\mathrm{x}, \mathrm{y})$.

Let, $\quad X=\left\{x_{i} \mid i=1,2, \ldots N\right\}$ and $Y=\left\{y_{i} \mid i=1,2, \ldots N\right\}$ be the original and test image signal respectively.

Then, Structural Similarity (SSIM) Index between two image signals x \& y will be given as:

$$
\operatorname{SSIM}(X, Y)=\frac{\left(2 \mu_{x} \mu_{y}+c_{1}\right)\left(2 \sigma_{x y}+c_{2}\right)}{\left(\mu_{x}^{2}+\mu_{y}^{2}+c_{1}\right)\left(\sigma_{x}^{2}+\sigma_{y}^{2}+c_{2}\right)} .
$$

Constants $\mathrm{C}_{1}, \mathrm{C}_{2}$, are used in equations 5 to avoid unstability when denominators $\left(\mu_{x}{ }^{2}+\mu_{y}{ }^{2}\right)$ and $\left(\sigma_{x}^{2}+\sigma_{y}^{2}\right)$ approach to zero. where, $C_{1}=\left(K_{1} L\right)^{2}, C_{2}=\left(K_{2} L\right)^{2}$.

$\mathrm{L}=$ dynamic range of the pixel values (255 for 8-bit gray scale image) and $\mathrm{K}_{1}, \mathrm{~K}_{2}$ are very small positive constants such that $\mathrm{K}<<1$.

They applied their quality measurement method to local region using a sliding window approach. Starting from top-left corner of the image, a sliding window of size $8 \times 8$ moves pixel by pixel horizontally and vertically through all the row and column of the image until the bottom right corner is reached. The overall image quality is obtained by computing the average of SSIM values over all the windows. If $M$ is total number of windows then mean structural similarity index is given as:

$$
\operatorname{MSSIM}(X, Y)=\frac{1}{M} \sum_{j=1}^{M} \operatorname{SSIM}\left(x_{j}, y_{j}\right)
$$

\subsection{Visual Information Fidelity Criterion in Pixel Domain (VIFP)}

The visual information fidelity in pixel domain (VIFP) is derived from a quantification of two mutual information quantities: the mutual information between the input and the output of the HVS channel when no distortion channel is present and the mutual information between the input of the distortion channel and the output of the HVS channel for the test image. Assumption was made that, in the absence of any distortions, this signal passes through the HVS channel of a human observer before entering the brain, which extracts cognitive information from it. For distorted images, it was assumed that the reference signal has passed through another distortion channel before entering the HVS. Combining these two quantities, a visual information fidelity measure for IQA is derived. But, for distortion types that are significantly different from blur and white noise, such as JPEG compression, the model fails to reproduce the perceptual annoyance adequately and also to implement VIFP criterion in, a number of assumptions are needed about the source, distortion, and HVS models.

\section{COLOR IMAGE QUALITY}

The human visual system can distinguish thousands of different colour shades and intensities, but only around 100 shades of gray. Therefore, in an image, a great deal of extra information may be contained in the color, and this extra information can then be used to simplify image analysis, e.g. object identification and extraction based on colour. Although, mechanisms of color processing by human brain is not completely understood. 


\subsection{Attributes of Color}

Three attributes are used to distinguish one color from another. First attribute is hue which measures dominant wavelength in a mixture of light waves or wavelength of the pure colour observed in the signal. Second attribute is saturation which gives the relative purity of color or the amount of white light mixed with the hue. Third attribute is brightness which is the attribute of a visual sensation according to which an area appears to emit more or less light. It distinguishes the gray levels. Hue and saturation taken together is called chrominance. Thus, any color can be characterized by its brightness and chrominance. In this paper chrominance information is extracted from HSI model of image and this information is used to assess the quality.

\subsection{HSI Color Model}

Color models/spaces facilitate the specification of colors following some standard way. RGB and CMY color models are well suited for hardware implementations; these models do not fit well human perception of colors. Also, color images cannot be thought of as being composed of three primary images that combine to form a single image. HSI model has unique advantage that it decouples the intensity component from the color information (hue and saturation) in a color image.

So HSI is an ideal tool for developing image processing algorithms based on color descriptions [15] that are natural and intuitive to humans, who are the ultimate users of these algorithms. So, it can be concluded that RGB is ideal for image color generation but its use for color description is limited. Where, HSI model is used for Color image manipulation. So, RGB image is converted in to corresponding HSI image for quality evaluation.

Important components of the HSI model are the vertical intensity axis (I), the length of the vector to the color point which represents saturation (S), and the angle of that vector $(\theta)$ which represents hue $(\mathrm{H})$ as shown in color triangle shown in figure 1 .

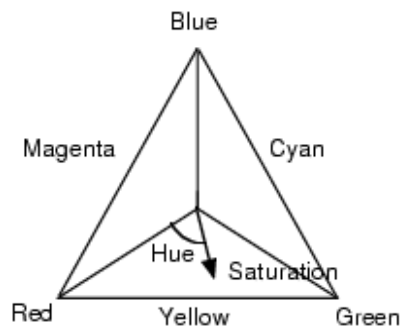

Figure 1. HSI Color Triangle

\section{PROPOSED COLOR IMAGE QUALITY INDEX ( $\left.\mathbf{Q}_{\text {new }}\right)$}

Measurement of visual quality is of fundamental importance to numerous image processing applications. Any imaging system can use the quality metric to adjust itself automatically for obtaining improved quality images. It can be used to compare and evaluate various image processing systems and algorithms.

A new quality Index in spatial domain is proposed to assess the quality of various noise contaminated, compressed and blurred images. Image noise is undesirable by product of the image capture. The principal sources of noise in images arise during image acquisition and transmission. Gaussian noise arises in an image due to factors such as electronic circuit noise and sensor noise due to poor illumination and/or high temperature. Speckle noise is caused by errors in data transmission. This kind of noise generally affects the ultrasound images and radar images. Salt and Pepper noise is found in situations, where quick transients such as faulty switching take place during imaging. It is found generally in mammogram images. Blur is introduced due to motion that is either due to the motion of the object being imaged or by the motion of imaging system.

A new index is proposed by modelling any image distortion as a combination of factors like luminance, structure, edge and chrominance. The proposed quality metric is defined as:

$\boldsymbol{Q}_{\text {new }}=\left[(\mathrm{Lum}) \times(\boldsymbol{S C}) \times(\boldsymbol{E S}) \times\left(C_{r}\right)\right]$

where,

Lum is the luminance comparison function $S C$ is the structure correlation of the two images $E S$ represents the edge similarity of the two images $C_{r}$ represents color similarity of two images

Let, $\quad X=\left\{x_{i} \mid i=1,2, \ldots N\right\}$ and $Y=\left\{y_{i} \mid i=1,2, \ldots N\right\} \quad$ be orignal and test (distorted one) image signals respectively. The statistical features are measured locally starting from top left corner of the image, a sliding window of size $\mathrm{NxN}$ where $\mathrm{N}=8$ is moved pixel by pixel horizontally and vertically through all the rows and columns of image until the bottom right corner is reached.

Local luminance of each signal is estimated by the mean intensity $\mu_{x}, \mu_{y}$.

$$
\text { Lum }=l\left(\mu_{x}, \mu_{y}\right)=\frac{2 \mu_{x} \mu_{y}}{\left(\mu_{x}^{2}+\mu_{y}^{2}\right)}
$$

Structural correlation (SC) of two images is determined by using Pearson's correlation coefficient given as:

$$
S C=\frac{\sum_{i=1}^{N}\left(x_{i}-\mu_{x}\right)\left(y_{i}-\mu_{y}\right)}{\sqrt{\left(\sum_{i=1}^{N}\left(x_{i}-\mu_{x}\right)^{2}\right)\left(\sum_{i}^{N}\left(y_{i}-\mu_{y}\right)^{2}\right)}}
$$

At each step, local statistics like standard deviation $\sigma_{x}, \sigma_{y}$, and covariance $\sigma_{x y}$ are computed with in local window.

Edges in images are the areas with strong intensity contrast [13-15]. Edge degradations are due to discontinuities in edge, decrease of edge sharpness, missing edge points etc. Sobel operator is used for edge detection; both horizontal and vertical edge mask is used for 2-D spatial gradient measurement in $\mathrm{R}, \mathrm{G}$, and $\mathrm{B}$ vector space individually. Edges are to be computed in RGB vector space directly which can be calculated by finding the gradient of the vector $\mathbf{c}$ which is an arbitrary vector in RGB color space given as:

$$
\boldsymbol{c}=\left[\begin{array}{l}
C_{R} \\
C_{G} \\
C_{B}
\end{array}\right]=\left[\begin{array}{l}
R \\
G \\
B
\end{array}\right]
$$

Edges are to be computed in RGB vector space directly which can be calculated by finding the gradient of the vector $\mathbf{c}$. Computation will be done as follows:

- Compute the $\mathrm{x}$ and $\mathrm{y}$ derivative of three component images R, G, B using sobel operators i.e.

$$
\frac{\partial R}{\partial x}, \frac{\partial R}{\partial y}, \frac{\partial G}{\partial x}, \frac{\partial G}{\partial y}, \frac{\partial B}{\partial x} \text { and } \frac{\partial B}{\partial y}
$$


- Let $\mathrm{r}$, g, and $\mathrm{b}$ be the unit vectors along the R, G, B axis of RGB color space and define the vectors:

$$
\begin{gathered}
u=\frac{\partial R}{\partial x} \boldsymbol{r}+\frac{\partial G}{\partial x} \boldsymbol{g}+\frac{\partial B}{\partial x} \boldsymbol{b} \\
v=\frac{\partial R}{\partial y} \boldsymbol{r}+\frac{\partial G}{\partial y} \boldsymbol{g}+\frac{\partial B}{\partial y} \boldsymbol{b}
\end{gathered}
$$

- Take the dot product of the vectors computed in previous step to find:

$$
\begin{aligned}
& G_{x x}=u \cdot u=\left|\frac{\partial R}{\partial x}\right|^{2}+\left|\frac{\partial G}{\partial x}\right|^{2}+\left|\frac{\partial B}{\partial x}\right|^{2} \ldots \\
& G_{y y}=v \cdot v=\left|\frac{\partial R}{\partial y}\right|^{2}+\left|\frac{\partial G}{\partial y}\right|^{2}+\left|\frac{\partial B}{\partial y}\right|^{2} \\
& G_{x y}=u \cdot v=\frac{\partial R}{\partial x} \frac{\partial R}{\partial y}+\frac{\partial G}{\partial x} \frac{\partial G}{\partial y}+\frac{\partial B}{\partial x} \frac{\partial B}{\partial y}
\end{aligned}
$$

- Color image gradient is given by calculating direction of maximum rate of change of vector $\mathrm{c}(\mathrm{x}, \mathrm{y})$ at coordinates $(\mathrm{x}, \mathrm{y})$ calculated as:

$$
\theta(x, y)=\frac{1}{2} \tan ^{-1}\left[\frac{2 G_{x y}}{\left(G_{x x}-G_{y y}\right)}\right]
$$

- Magnitude of the gradient in that direction $\theta(\mathrm{x}, \mathrm{y})$ is given by:

$$
G_{1}=\left\{\frac{1}{2}\left[(a 1)+(a 2) \cos 2 \theta+2 G_{x y} \sin 2 \theta\right]\right\}^{1 / 2} \ldots
$$

$$
\text { where, } a 1=\left(G_{x x}-G_{y y}\right), a 2=\left(G_{x x}-G_{y y}\right)
$$

- Similarly magnitude of the gradient $\left(G_{2}\right)$ is computed for distorted image.

- Edge similarity (ES) of two images is given as:

$$
E S=\frac{2 G_{1} G_{2}}{\left[\left(G_{1}{ }^{2}\right)+\left(G_{2}{ }^{2}\right)\right]}
$$

Chroma $\left(\mathrm{C}_{\mathrm{r}}\right)$ is indicating the chrominance information. Hue and saturation both are two important attributes of color that represents the chrominance (color) information. So, to calculate chrominance information there is need to convert RGB image in to HSI. Hue and Saturation information together represent color information within the image. Color information is calculated for both original and distorted image. Finally, color similarity (Chroma) of two images is calculated.
The block diagram of the Proposed Quality Assessment Model is shown in figure 2 .

\section{RESULTS \& DISCUSSION}

In order to evaluate the performance of proposed quality index $\mathrm{Q}_{\text {new }}$, experiments have been done on standard colored test image available in TID 2008 database [16]. This quality index is applied to images using a sliding window approach, with a window size of $8 \times 8$, starting from the top left corner until bottom right corner is reached.

The original 24-bit color $512 \times 384$ "house" test image distorted by a wide variety of corruptions is shown in figure 3 .

Distortions that are considered are shown in table 1 which are additive gaussian noise, additive noise in color components, impulsive salt-pepper noise, blurring, JPEG compression, JPEG 2000 compression, JPEG transmission error, JPEG 2000 transmission error.

Table 1. Different distortions used

\begin{tabular}{|l|l|l|}
\hline $\begin{array}{l}\text { Sr. } \\
\text { No. }\end{array}$ & $\begin{array}{l}\text { Type of distortion } \\
\text { (four levels for each } \\
\text { distortion) }\end{array}$ & $\begin{array}{l}\text { Correspondence to } \\
\text { practical situation }\end{array}$ \\
\hline 1. & Additive Gaussian noise & Image acquisition \\
\hline 2. & $\begin{array}{l}\text { Additive noise in color } \\
\text { components }\end{array}$ & Image acquisition \\
\hline 3. & Gaussian blur & Image registration \\
\hline 4. & Impulse noise & Image acquisition \\
\hline 5. & JPEG compression & JPEG compression \\
\hline 6. & JPEG2000 compression & JPEG2000 compression \\
\hline 7. & JPEG transmission errors & Data transmission \\
\hline 8. & $\begin{array}{l}\text { JPEG2000 transmission } \\
\text { errors }\end{array}$ & Data transmission \\
\hline
\end{tabular}

The proposed quality index $\left(\mathrm{Q}_{\text {new }}\right)$ has been implemented on the standard colored house image taken from TID 2008 database. All of the corrupted images as shown in fig. 3(b)-(i) have been subjected to different quality measures like MSE, PSNR [4], MSSIM [6], VIFP [10], SHSIM [12] and proposed $\mathrm{Q}_{\text {new }}$ to prepare the performance comparison of quality assessment metrics. Quality will also be evaluated for different levels of distortions for each quality metric.

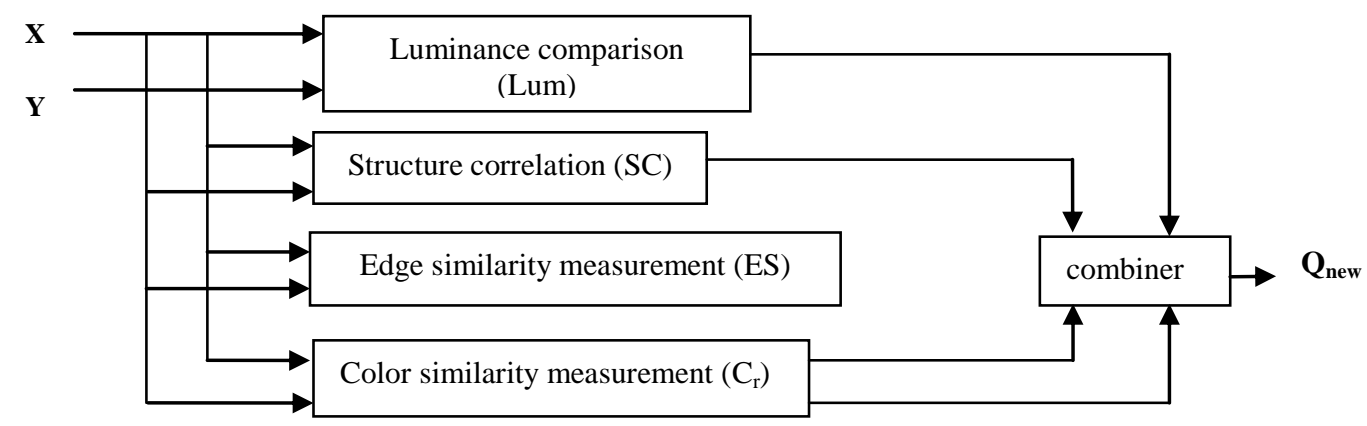

Figure 2. Block Diagram of Proposed Quality Assessment Model 


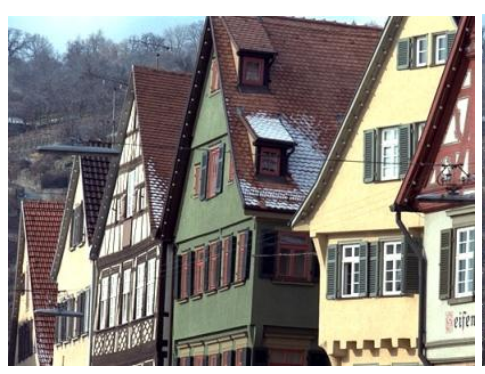

(a) original house image

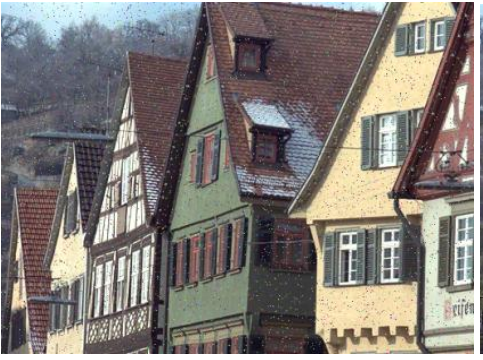

(b) impulse noise image

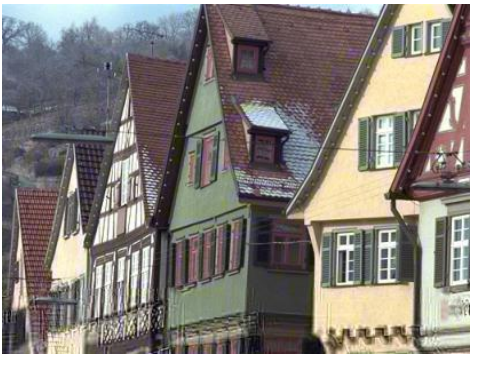

(c) JPEG transmission error

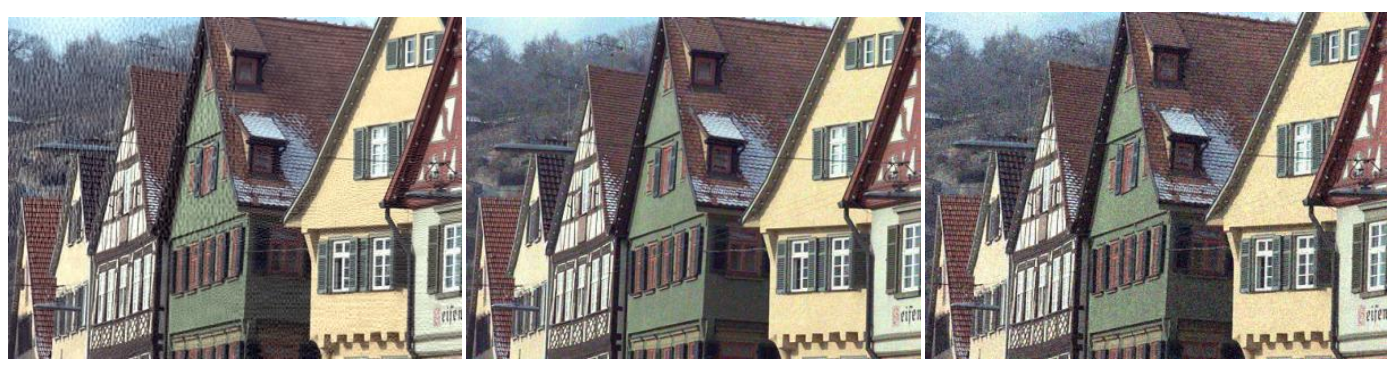

(d)JPEG2000 transmission error (e) gaussian noise in color components

(f) Gaussian noise

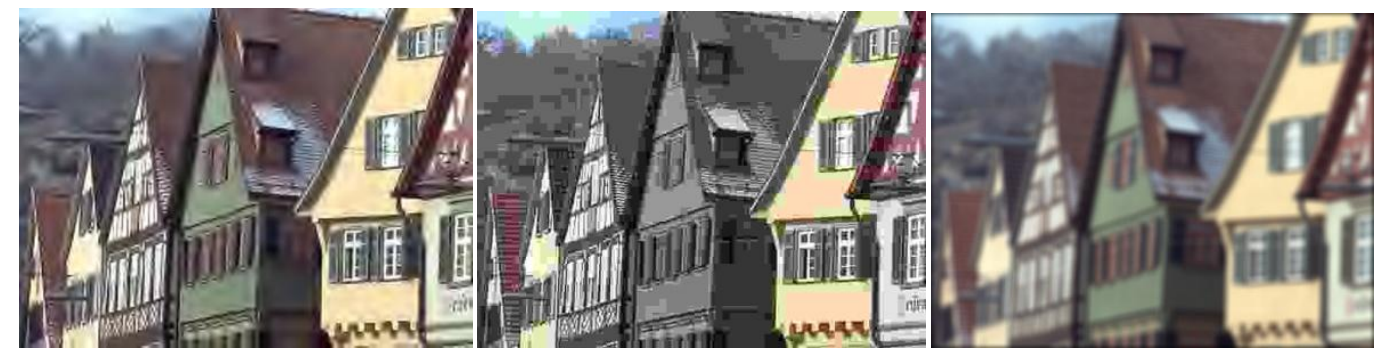

(g) JPEG2000 compressed image

(h) JPEG compressed image

(i) Blurred image

Figure 3 Quality assessment of standard 24-bit color $512 \times 384$ house image corrupted by different distortions

Table 2. Quality assessment results of structure hue similarity index (SHSIM), visual information fidelity at pixel domain (VIFP) at different levels of distortions

\begin{tabular}{|c|c|c|c|c|c|c|c|c|}
\hline \multicolumn{10}{|c|}{ SHSIM } \\
\hline Level & $\begin{array}{c}\text { Gaussian } \\
\text { blur }\end{array}$ & $\begin{array}{c}\text { JPEG } \\
\text { compression }\end{array}$ & $\begin{array}{c}\text { JPEG2000 } \\
\text { compression }\end{array}$ & $\begin{array}{c}\text { Additive } \\
\text { Gaussian } \\
\text { noise }\end{array}$ & $\begin{array}{c}\text { additive noise } \\
\text { in color } \\
\text { components }\end{array}$ & $\begin{array}{c}\text { JPEG2000 } \\
\text { transmission } \\
\text { errors }\end{array}$ & $\begin{array}{c}\text { JPEG } \\
\text { transmission } \\
\text { errors }\end{array}$ & $\begin{array}{c}\text { Impulse } \\
\text { noise }\end{array}$ \\
\hline $\mathbf{1}$ & 0.5726 & 0.5477 & 0.5518 & 0.5247 & 0.5236 & 0.5659 & 0.5656 & 0.5816 \\
\hline $\mathbf{2}$ & 0.5668 & 0.5299 & 0.5460 & 0.5170 & 0.5160 & 0.5624 & 0.5655 & 0.5799 \\
\hline $\mathbf{3}$ & 0.5570 & 0.5175 & 0.5404 & 0.5102 & 0.5100 & 0.5499 & 0.5194 & 0.5767 \\
\hline $\mathbf{4}$ & 0.5409 & 0.4903 & 0.5243 & 0.5038 & 0.5032 & $\mathbf{0 . 5 5 5 8}$ & $\mathbf{0 . 5 5 8 6}$ & 0.5704 \\
\hline
\end{tabular}

\begin{tabular}{|c|c|c|c|c|c|c|c|c|}
\hline \multicolumn{10}{|c|}{ VIFP } \\
\hline $\begin{array}{c}\text { Leve } \\
1\end{array}$ & $\begin{array}{c}\text { Gaussian } \\
\text { blur }\end{array}$ & $\begin{array}{c}\text { JPEG } \\
\text { compression }\end{array}$ & $\begin{array}{c}\text { JPEG2000 } \\
\text { compression }\end{array}$ & $\begin{array}{c}\text { Additive } \\
\text { Gaussian } \\
\text { noise }\end{array}$ & $\begin{array}{c}\text { additive noise } \\
\text { in color } \\
\text { components }\end{array}$ & $\begin{array}{c}\text { JPEG2000 } \\
\text { transmission } \\
\text { errors }\end{array}$ & $\begin{array}{c}\text { JPEG } \\
\text { transmission } \\
\text { errors }\end{array}$ & $\begin{array}{c}\text { Impuls } \\
\text { e noise }\end{array}$ \\
\hline $\mathbf{1}$ & 0.8736 & 0.8991 & 0.8936 & 0.8784 & 0.9033 & 0.6922 & 0.9010 & 0.8596 \\
\hline $\mathbf{2}$ & 0.7956 & 0.8395 & 0.8557 & 0.8530 & 0.8956 & $\mathbf{0 . 8 9 8 8}$ & 0.8835 & 0.8098 \\
\hline $\mathbf{3}$ & 0.6133 & 0.6522 & 0.8005 & 0.8084 & 0.8820 & $\mathbf{0 . 7 4 8 6}$ & 0.8682 & 0.7486 \\
\hline $\mathbf{4}$ & 0.3688 & 0.4825 & 0.5075 & 0.7480 & 0.8528 & 0.4276 & 0.7325 & 0.6573 \\
\hline
\end{tabular}


Table 3. Quality assessment results of mean structural similarity index (MSSIM), and Proposed index $\left(Q_{\text {new }}\right)$ at different levels of distortions

\begin{tabular}{|c|c|c|c|c|c|c|c|c|}
\hline \multicolumn{10}{|c|}{ MSSIM } \\
\hline Level & $\begin{array}{c}\text { gaussian } \\
\text { blur }\end{array}$ & $\begin{array}{c}\text { JPEG } \\
\text { compression }\end{array}$ & $\begin{array}{c}\text { JPEG2000 } \\
\text { compression }\end{array}$ & $\begin{array}{c}\text { Additive } \\
\text { Gaussian } \\
\text { noise }\end{array}$ & $\begin{array}{c}\text { additive noise } \\
\text { in color } \\
\text { components }\end{array}$ & $\begin{array}{c}\text { JPEG2000 } \\
\text { transmission } \\
\text { errors }\end{array}$ & $\begin{array}{c}\text { JPEG } \\
\text { transmission } \\
\text { errors }\end{array}$ & $\begin{array}{c}\text { Impulse } \\
\text { noise }\end{array}$ \\
\hline $\mathbf{1}$ & 0.9653 & 0.9668 & 0.9664 & 0.9668 & $\mathbf{0 . 9 6 6 9}$ & 0.9655 & 0.9668 & 0.9655 \\
\hline $\mathbf{2}$ & 0.9631 & 0.9667 & 0.9661 & 0.9666 & $\mathbf{0 . 9 6 6 9}$ & 0.9622 & 0.9665 & 0.9635 \\
\hline $\mathbf{3}$ & 0.9566 & 0.9656 & 0.9657 & 0.9664 & 0.9666 & $\mathbf{0 . 9 6 5 1}$ & 0.9662 & 0.9603 \\
\hline $\mathbf{4}$ & 0.9417 & 0.9637 & 0.9610 & 0.9653 & 0.9662 & 0.9558 & 0.9534 & 0.9535 \\
\hline
\end{tabular}

\begin{tabular}{|c|c|c|c|c|c|c|c|c|}
\hline \multicolumn{9}{|c|}{$\mathbf{Q}_{\text {new }}$} \\
\hline Level & $\begin{array}{c}\text { Gaussian } \\
\text { blur }\end{array}$ & $\begin{array}{c}\text { JPEG } \\
\text { compression }\end{array}$ & $\begin{array}{c}\text { JPEG2000 } \\
\text { compression }\end{array}$ & $\begin{array}{c}\text { Additive } \\
\text { Gaussian } \\
\text { noise }\end{array}$ & $\begin{array}{c}\text { additive noise } \\
\text { in color } \\
\text { components }\end{array}$ & $\begin{array}{c}\text { JPEG2000 } \\
\text { transmission } \\
\text { errors }\end{array}$ & $\begin{array}{c}\text { JPEG } \\
\text { transmission } \\
\text { errors }\end{array}$ & $\begin{array}{c}\text { Impulse } \\
\text { noise }\end{array}$ \\
\hline $\mathbf{1}$ & 0.8001 & 0.6190 & 0.6437 & 0.4378 & 0.4336 & 0.7581 & 0.7614 & 0.8857 \\
\hline $\mathbf{2}$ & 0.6534 & 0.4452 & 0.5583 & 0.3879 & 0.3855 & 0.7354 & 0.7315 & 0.8385 \\
\hline $\mathbf{3}$ & 0.3860 & 0.3924 & 0.4629 & 0.3495 & 0.3615 & 0.5825 & 0.6766 & 0.7563 \\
\hline $\mathbf{4}$ & 0.1717 & 0.2524 & 0.2726 & 0.3180 & 0.3318 & 0.4184 & 0.4284 & 0.6234 \\
\hline
\end{tabular}

Figure 3 is showing house images with different distortions at level 4. However, performance of proposed index and other existing metrics will also be checked at all level of distortions i.e. level 1 (minimum distortion), level 2, level 3, level 4 (maximum distortion). It is clear that with increase in the distortion from level 1 to level 4, there must be decrease in the quality. However, from table 1 and table 2 it is clear that MSSIM, SHSIM and VIFP values are decreasing with increase in distortion but at some points quality values are totally inconsistent showing increase in quality with increase in the level of distortion. These inconsistent values are shown by bold values. But Proposed index $\left(\mathrm{Q}_{\text {new }}\right)$ values as shown in table 3 are decreasing with increase in the level of distortion, across various types of distortions.
Quality metrics MSE, PSNR, MSSIM, VIFP, SHSIM and $\mathrm{Q}_{\text {new }}$ are HVS based similarity measures. While assessing the quality, original image is compared with distorted one. In case of original image 3(a) values of these metrics is maximum i.e. unity since it is completely undistorted image. MSE will be zero and PSNR will be infinite. The order of quality according to HVS is that highly blurred image must be of less quality than compressed image and compressed image must be of less quality than gaussian contaminated images and gaussian contaminated image is of less quality than transmission error contaminated one and finally impulse noise contaminated image will be of highest quality. So, across various distortions from 3(b) to 3(i) quality must decrease accordance to HVS.

Table 4 Comparative quality measurement of House image with different types of distortions at distortion level 4

\begin{tabular}{|c|c|c|c|c|c|c|c|}
\hline \multirow{2}{*}{$\begin{array}{c}\text { Fig. } \\
\text { No. }\end{array}$} & Type of distortion & \multicolumn{5}{|c|}{ Quality Metrics } \\
\hline 3(a) & Orignal image & 1 & 1 & 1 & 1 & Infinite & 0 \\
\hline 3(b) & Impulse noise & 0.6234 & 0.5704 & 0.6573 & 0.9535 & 37 & 143.85 \\
\hline 3(c) & JPEG transmission errors & 0.4284 & 0.5586 & 0.7325 & 0.9534 & 38.1 & 97.03 \\
\hline 3(d) & JPEG2000 transmission errors & 0.4184 & 0.5558 & 0.4276 & 0.9558 & 38 & 102.6 \\
\hline 3(e) & Additive noise in color components & 0.3318 & 0.5032 & 0.8528 & 0.9662 & 38.5 & 80.85 \\
\hline 3(f) & Additive Gaussian noise & 0.3180 & 0.5038 & 0.7480 & 0.9653 & 38.4 & 86.10 \\
\hline 3(g) & JPEG2000 compression & 0.2726 & 0.5243 & 0.5075 & 0.9610 & 38.4 & 85.48 \\
\hline 3(h) & JPEG compression & 0.2524 & 0.4903 & 0.4825 & 0.9637 & 38.1 & 98.416 \\
\hline $\mathbf{3 ( i )}$ & Gaussian blur & 0.1717 & 0.5409 & 0.3688 & 0.9417 & 37.6 & 124.59 \\
\hline
\end{tabular}




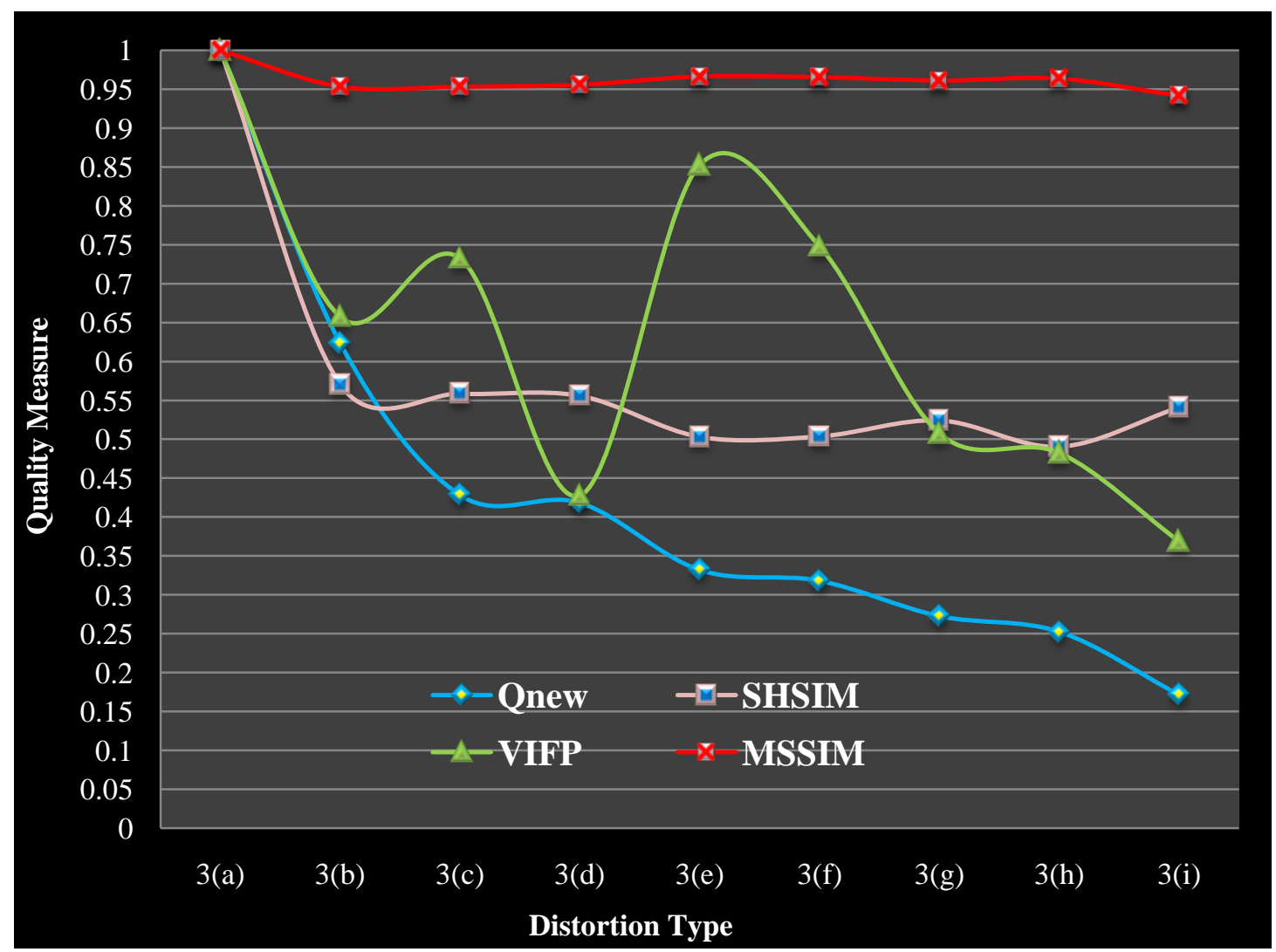

Figure 4. Performance comparison of quality assessment metrics over house images with different types of distortion

From table 4 it is clear that, MSE \& PSNR results are fully inconsistent with HVS since PSNR quality values are same across various distortion types although they are having different visual perception. Where, MSE values must decrease from 3(b) to 3(i) according to HVS. But MSE results are completely inconsistent.

Figure 4 and table 4 shows that proposed index $\left(\mathrm{Q}_{\text {new }}\right)$ working accurately since its values is decreasing from 3(b) to 3(i). But structure \& hue similarity index (SHSIM) results are not consistent with HVS since the quality sequence is not followed by it. SHSIM is showing blurred image 3(i) to be of higher quality than gaussian noise contaminated image 3(f) which is totally in consistent with HVS. Also, it is showing JPEG 2000 compressed image $3(\mathrm{~g})$ to be of more quality than gaussian contaminated one 3(f) \& 3(e) which is not accordance to HVS. Also, Results of visual information fidelity (VIFP) are completely violating HVS in case of JPEG 2000 transmission showing, very less value of quality even smaller than JPEG and JPEG 2000 compression. Other inconsistent values of VIFP are at additive noise 3(e-f), and JPEG transmission error 3 (c).

Similarly Results of MSSIM are completely violating HVS in case of JPEG compression 3(h) and JPEG 2000 compression $3(\mathrm{~g})$ showing, maximum value of quality. Also MSSIM values are not following HVS based sequence of quality across other distortions. Inconsistent values of MSSIM values are at JPEG compression 3(d), additive Gaussian noise 3(e-f), JPEG 2000 compression 3(h) and JPEG compression 3(g).

\section{CONCLUSION \& FUTURE SCOPE}

The intention of this project work is to understand both the fundamental philosophies and the basic approaches of image quality assessment algorithms. Three types of knowledge are taken in to consideration in the design of image quality assessment methods: knowledge about the human visual system (HVS); knowledge about high-quality images; and knowledge about image distortions. Thus, a number of issues would need to be addressed to carry out image quality assessment which includes availability of the reference images, the required level of quality, application scope (general-purpose or application-specific), application goal (for quality monitoring, system benchmarking, or algorithm optimization), and speed requirements (e.g., real-time or offline).

Experiments performed, on standard color images for wide variety of distortions indicate that proposed new color image quality metric $Q_{\text {new }}$ in spatial domain is overcoming the limitation of existing quality methods discussed in the paper. It is concluded that, MSE and PSNR values are showing very poor correlation with HVS. MSSIM, VIFP and SHSIM values are also not showing full consistency with HVS across various distortions discussed. However, $\mathrm{Q}_{\text {new }}$ values are following desired HVS sequence of quality across various distortions as well as showing the consistent results at different levels of distortions.

The application scope includes, but is not limited to, image compression, communication, acquisition, display, restoration, enhancement, segmentation, detection, and classification of photographic images, medical images, geographic images, satellite images, and the astronomical images. But to achieve the best image quality evaluation for these specific applications, there is still a lot more work to do. Color image quality assessment can be further extended to video quality assessment. 


\section{REFERENCES}

[1] Thung, Kim-Han, Raveendran, Paramesran. 2009. A survey of image quality measures. In Proceedings of International Conference for Technical Postgraduates (TECHPOS), pp.1-4.

[2] D. A. Silverstein and J. E. Farrell. 1996. The relationship between image fidelity and image quality. In Proceedings of IEEE International Conference on Image Processing, pp. 881-884.

[3] B.Girod. 1993. What's wrong with mean-squared error. In Digital Images and Human Vision (A.B.Watson, ed.), pp. 207-220.

[4] Z. Wang and A. C. Bovik. 2009. Mean squared error: love it or leave it? - A new look at signal fidelity measures. IEEE Signal Processing Magazine, vol. 26, no. 1, pp. 98-117, Jan. 2009.

[5] Z. Wang and A. C. Bovik. 2002. A universal image quality index," IEEE Signal Processing Letters, vol. 9, pp. 81-84.

[6] Z. Wang, A. C. Bovik, H. R. Sheikh, and E. P. Simoncelli. 2004. Image quality assessment: From error measurement to structural similarity," IEEE Transaction on Image Processing, vol. 13, pp. 600-612.

[7] Z. Wang, A. C. Bovik, and E. P. Simoncelli. 2005. Structural Approaches to Image Quality Assessment. Handbook of Image and Video Processing, $2^{\text {nd }}$ ed., Academic Press, pp. 1-33.

[8] Ho-Sung Han, Dong-O Kim, Rae-Hong Park. 2009. Structural Information-Based Image Quality Assessment Using LU Factorization. IEEE Signal Processing Letters, Vol. 55, No. 1, pp. 165-171.
[9] Alan C. Brooks, Xiaonan Zhao, and Thrasyvoulos N. Pappas. 2008. Structural Similarity Quality Metrics in a Coding Context: Exploring the Space of Realistic Distortions. IEEE Transactions on image processing, Vol. 17, No. 8, pp.1261-1263.

[10] Hamid R. Sheikh and Alan C. Bovik. 2006. Image information and visual quality. IEEE Transactions on Image Processing, Vol.15, No.2, pp. 430-444.

[11] D.J. Granrath. 2005. The role of human visual models in image processing. IEEE Transactions on image processing, Vol. 69, No. 5, pp. $552-561$.

[12] Y. Shi, Y. Ding, R. Zhang, Jun Li. 2009. Structure and Hue Similarity for Color Image Quality Assessment. In Proceedings of International Conference on Electronic Computer Technology, pp. 329 - 333.

[13] W.Fu, G. Xiaodong, Y. Wang. 2008. Image Quality Assessment Using Edge and Contrast Similarity. In Proceedings of International joint conference on Neural Networks, pp.852-855.

[14] Z. Wang and Alan C. Bovik. 2006. Handbook of Modern Image Quality Assessment, Morgan \& Claypool, pp. 1-65.

[15] Rafael C. Gonzalez and Richards E. Woods. 2006 Digital Image Processing, second edition, Pearson Prentice Hall, pp.304-364.

[16] N. Ponomarenko, V. Lukin, K. Egiazarian, J. Astola, M. Carli, and F. Battisti. 2008. Color image database for evaluation of image quality metrics. IEEE 10th Workshop on Multimedia Signal Processing, pp.403408 